\title{
Fast Segmentation of Foreign Fiber Image
}

\author{
Yutao Wu, Wenzhu Yang, Zhenbo Li, and Daoliang $\mathrm{Li}^{*}$ \\ College of Information and Electrical Engineering, \\ China Agricultural University, Beijing 100083, P.R. China \\ dliangl@cau.edu.cn
}

\begin{abstract}
In the textile industry, different types of foreign fibers may be mixed in cotton, and the foreign fibers seriously affect the quality of cotton products. The step of image segmentation is of vital importance in the process of the foreign fibers identification, which is, in the same way, the foundation for cotton foreign fiber automated inspection. This paper presents a new approach for fast segmentation of foreign fiber images. This approach includes four main steps, i.e., image transformation, image block, image background extraction, image enhancement and segmentation. In the first step, we transform the captured color images into gray-scale images, and invert the color of the transformed images. In the second step, the proportion relationship between target image and background was analyzed, and then the whole foreign fibers image was divided into several blocks based on the analysis results. In the third step, the background of foreign fiber image was extracted by image corrosion and gray-level correction. In the final step, the histogram of the gray-scale image was analyzed, and a piecewise linear transform model was proposed to enhance the image blocks based on the analysis results, and then the image blocks were segmented by Otsu's method. The experiment results indicate that the proposed method can segment the foreign fiber image directly and precisely, and the speed of image processing is much faster than that of the conventional methods.
\end{abstract}

Keywords: Cotton, Foreign fibers, Fast segmentation, image processing, image block, background extraction, Ostu method.

\section{Introduction}

The foreign fibers in cotton refer to those non-cotton fibers and dyed fibers, such as hairs, binding ropes, plastic films, candy wrappers, and polypropylene twines, etc. Foreign fibers mixed with cotton during picking, storing, drying, transporting, purchasing and processing, are difficult to remove in spinning process, and can cause yarn breakage, even reducing the efficiency. Every low content of foreign fibers in cotton, especially in lint, will seriously affect the quality of the final cotton textile products, as they may debase the strength of the yarn, and are not easy to be dyed (Yang, et al., 2009). Since the price of the cotton for sale is affected by the content of

* Corresponding author. 
foreign fibers in it, the cotton farmers and traders are willing to keep the foreign fibers away to obtain a high price. This will lead to great economic loss for the cotton textile enterprises. Two main factors may lead to high level of foreign fiber content in cotton. One is the inappropriate picking technique. The foreign fibers are generally removed manually using human visual inspection, or mechanically using automated visual inspection (Lieberman et al., 1998; Su et al., 2006). When most western countries are using machines to pick up cotton automatically, Chinese cotton farmers in most regions are still picking cotton manually and putting them in polypropylene bags. Currently, foreign fibers are generally removed by hand picking methods using human visual inspection in most Chinese enterprises, which is time consuming and inefficient. So a machine vision system for online measurement of the content of foreign fibers in cotton is now being studied. High quality image acquisition, fast image processing, effective feature extraction, accurate object classification and precise content measurement are key factors in the implementation of the system (Li, et al., 2010).

The recognition of foreign fibers of targets is the key machine vision technology, in which image segmentation is an important step. Image segmentation is a process of partitioning an image into multiple regions and typically used to locate objects and boundaries (lines, curves, etc.). The aim of image segmentation is to partition the image into meaningful connected components to extract the features of the objects (Zhang et al., 2008). The segmentation results are the foundation of all subsequent image analysis and understanding, such as object representation and description, feature measurement, object classification, and scene interpretation, etc. Thus, throughout the image processing, image segmentation is an extremely important aspect. The popular approaches for image segmentation are: histogram-based methods, edge-based methods, region-based methods, model based methods and watershed methods (H. Zhang, J. E. Frittsb et al., 2008;Y. Jiang and Z.-H. Zhou et al., 2004).

Fast and precise segmentation has always been of great concern of people. Various image segmentation methods are reported in the literature (Bakker et al., 2008; Kim et al., 2003; Pichel et al., 2006) some of which are used in Automated Visual Inspection system in agriculture (Chen et al., 2002; Jiang et al., 2008). In recent years, researchers have developed more efficient, but also more complicated methods for segmentation. For example, Kainuller et al. (2007) used a statistical shape model combined with a constrained free-form model. Schmidt et al. (2007) presented a system that allows defining a set of rules, based on which abdominal organs are segmented (including the liver) using simple functions (like region-growing, or morphological operators). Liapis et al. (2004) proposed a wavelet-based algorithm for image segmentation based on color and texture properties. Furukawa et al. (2007) used a maximum posterior probability estimation for rough liver extraction subsequently refined with a level-set method. Seghers et al. (2007) presented an active shape model method, in which multiple local shape models are used. Susomboon et al. (2007) used intensity-based partition, texture-based classification, probability model, and thresholding to segment the liver.

The methods mentioned above may perform well in their specific circumstances, but it does not apply to segment images of cotton foreign fibers because of the low contrast. Due to the uneven thickness of the layers of cotton and foreign fibers of different colors and shapes, it is hard to attain a satisfying result by using the above conventional image segmentation methods. Therefore, we need to use the image pre-processing methods to eliminate the low image contrast and gray uniform of the background image. In the 
practice of image processing, image preprocessing refers to processing work in advance of the feature extraction, segmentation and matching of the image input. And the main aim of image preprocessing is to eliminate the irrelevant information in the origin image, regain the valuable and authentic information, and strengthen the detestability of relevant information, as well as to simplify date to the maximum, therefore to improve the reliability of feature extraction, segmentation, matching and recognition. In another words, image preprocessing provide better information base image for the image segmentation and makes it easy to image segmentation. In this article, a novel method based on image preprocessing and adaptive thresholding method is proposed to segment such low-contrast images.

In our research, the proportion relationship between target image and background was firstly analyzed, and then the whole foreign fibers image was divided into several blocks based on the analysis results. Thereafter, improve the contrast ratio by means of the background subtraction of each image block. In the next step, a piecewise linear enhancement model was established based on the results of the histogram analysis, and applied to enhance the contrast of the image further. Finally, the best thresholding was determined by the Otsu's method.

\section{$2 \quad$ Materials and Methods}

\subsection{Materials Preparation}

The foreign fibers used in this research were collected from cotton mills including feathers, hair, hemp rope, plastic films, polypropylene twine, colored thread, cloth piece etc. as shown in Fig. 1. Adequate pure lint with no foreign fibers was also

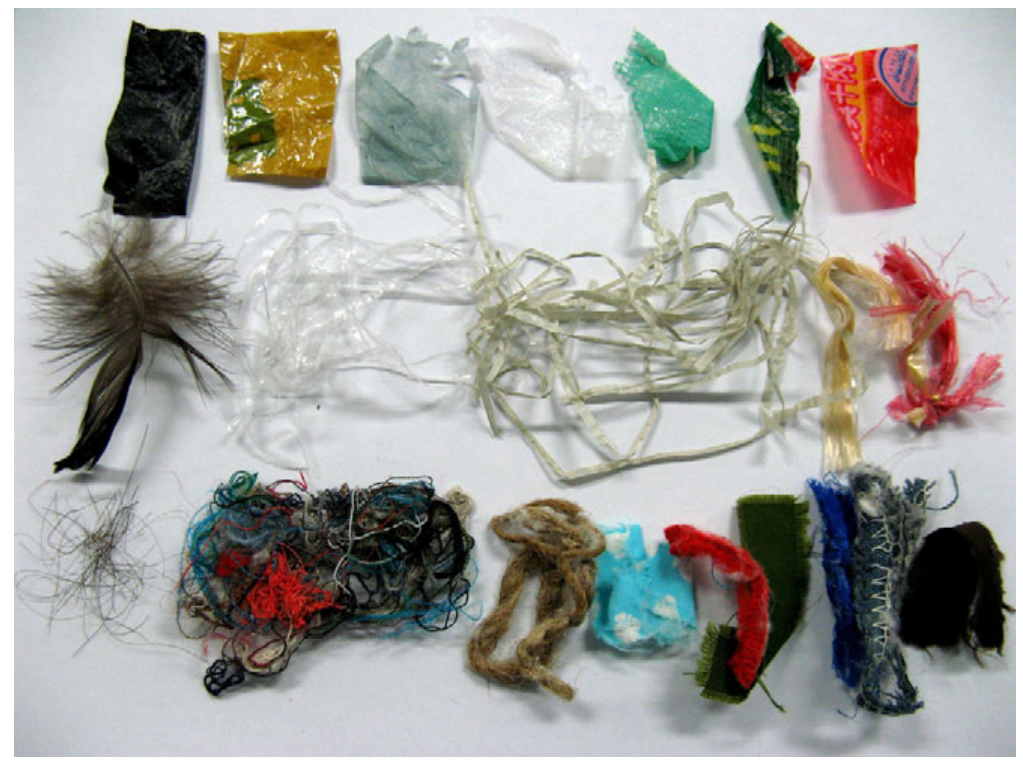

Fig. 1. Foreign fiber samples 
prepared for making the lint layer. The experiment selected a sufficient amount of lint cotton which does not include foreign fibers.

\subsection{Image Acquisition}

The Image Acquisition System has two cameras, two light sources, one shaft encoder, one synchronizing amplifier, two image acquisition boards and a computer, as shown in Figure 2. Colorful images are captured by a Canadian DALSA high-speed 3CCD color line scan camera under high-brightness LED lightning. The typical foreign fibers are concluded from the research on China's textile enterprises. To make the experiment easier, the foreign-fiber samples were dropping onto the surface of the pure lint one by one while the lint was feeding into the opening machine. After the lint with foreign fibers being opened, a continuous cotton layer is formed, $400 \mathrm{~mm}$ wide and $2 \mathrm{~mm}$ thick, Totally 40 color images with $4000 \times 500$ in resolution were then obtained.

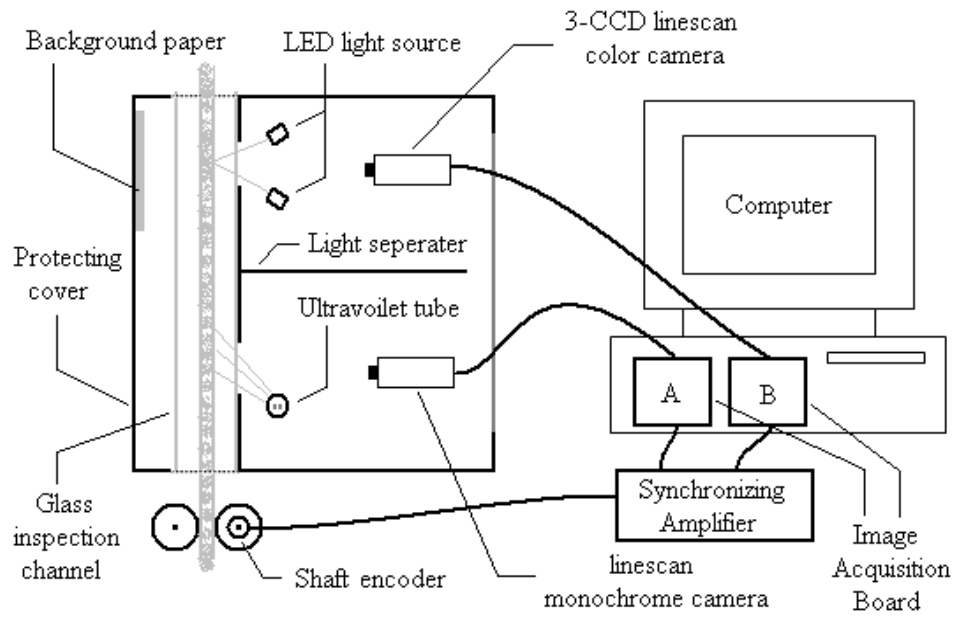

Fig. 2. The image acquisition system

By observing the images obtained, it was easy to find that the opened foreign fibers appeared in three typical forms, as shown in Fig. 3a-c, (1) sheet, such as plastic films, papers, etc., (2) wirelike, such as hair, color thread, etc., and (3) villiform, such as hemp rope, chemical fibers, etc.

As the original color image is too large but the target image is small, if the original image is directly inserted, it will result in an unclear target image, and affect the reader's vision. So same images in this paper are cut target images. 


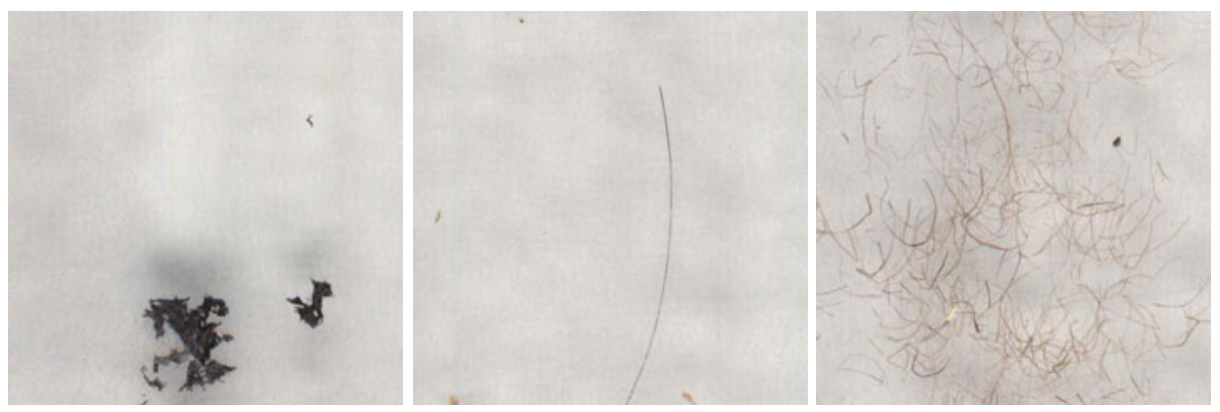

Fig. 3. Acquired color image examples: (a) opened plastic film, (b) opened hair, and (c) opened hemp rope

\subsection{Image Segmentation}

In the online detection of foreign fibers in lint using the machine-vision-based system, the speed and accuracy of algorithm are the key factors. Therefore, in order to ensure segmentation accuracy, the fastest segmentation algorithm should be chosen. Thresholding is a traditional method,which is widely used due to its computational simplicity, high speed and easy implementation. Ostu's Method, as an adaptive threshold method to confirm the threshold value, possesses the advantages of simple algorithm, high speed and so on. Moreover, it makes the biggest segmentation between-group variance implies getting the slightest chance to make errors, which means the Ostu's Method has the optimum segmentation threshold value so that the application of Ostu's method become widespread. However, due to the its problems existing in segmentation processing of images with heterogeneous background, small area proportion between object and background, or subtle difference of the gray rate between object and background, Otsu's Method has big variance in threshold value and therefore is unable to get segmented image with high quality. Nevertheless, cotton fiber image exactly belongs to the category small object image, which also has the characteristics such as small variance of gray rate and heterogeneous gray rate. Consequently, this paper employs preprocessing such as image block, image background extraction, gray-level correction, image enhancement, etc, to solve the problems.

\section{Image Blocking}

The segmentation result binarized by Otsu's method of an image is very satisfying for a good separation of figure and background. But, under the Otsu's method, only when object figure is larger than $25 \%$ of the whole image, the segmentation property is approximately optimum; when the area of moving object figure becomes smaller, the property of algorithm declines rapidly, which leads to smaller object figure and larger threshold value variance. Thus, to guarantee the segmentation accuracy and high-qualified segmentation result we divide the foreign fiber figure into several parts and combine them in sequence after image processing respectively, in order to resolve the problem of low ratio of the object figure and background area. 
In the analysis of the foreign fiber image, we finds that whole foreign fiber image is $4000 * 500$ large, with the object figure only occupying $5 \%$ or even less of the whole image. The most part of foreign fiber object figure occupies only $0.5 \%$ to $3 \%$ of the whole image. In order to increase the ratio of object figure and the background, we horizontally divide the origin foreign fiber image equally into 8 parts, each $500 * 500$.

\section{Image Background Extraction}

Previously stated, the contrast ratio of foreign fiber image is low, while the gray rate difference between the object and background is also very small. On the other hand, due to the uneven thickness of the layers of cotton, the gray rate of background of foreign fiber image is heterogeneous. Meanwhile, the segmentation effects of Otsu's method on the low-ratio image or heterogeneous gray rate image is unsatisfying. Cotton foreign fiber image is just the case mentioned above. In order to get qualified segmentation effect, we need to enhance the image contrast ration by the use of the method of background subtraction and to eliminate the impact from the heterogeneous image background.

Background subtraction is to, by means of mathematical morphology or image gray-level correction and other methods, get the image background without the object, then subtracted from the origin image so as to reach the aim to highlight the object and reduce the influence of image background. This paper processes the foreign fiber image by image corrosion in mathematical morphology to obtain the image background.

$f(x, y)$ is the tight gradation function of $L^{2}(R), g(i, j)$ is the gradation function for structural element.

The definition of corrosion for gradation function $f(x, y)$ is:

Corrosion definition:

$$
f \Theta g(x, y)=\min _{(i, j)}\{f(x+i, y+j)+g(i, j)\}
$$

To make calculation convenient, the gray structure elements often take 0 , that is $g(i, j)=0$. The structural element employed in the paper is round structural element with the radius of 3 . After the corrosion of foreign fiber image, we get a first-stage image background.

\section{Image Gray-Level Correction}

We can get background figure with comparative high quality by applying the above methods to the two kinds of foreign fiber image---wirelik and villiform, but we confront problems with the sheet foreign fiber image. The reason behind this is that the sheet foreign fiber image has larger object figure area, which incurs to the object figure remnants after the corrosion by radius 3 structural element. After the analyzing background figure of this type, we find that sheet foreign fiber image has greater gray-level and has more obvious difference with the cotton background. Therefore, we 
can improve the problems of this type via image gray-level correction to get highly-qualified background figure.

This paper utilized fixed threshold value 0.6 as a basic point to conduct image gray-level correction on image, that is, to replace the gray-level of all pixels that above 0.6 by certain needed gray-level value. The paper makes that certain value as 0 for convenience. Then we get a more satisfying background figure through image gray-level correction. Subsequently, subtracting origin foreign fiber image with the obtained background figure, we get the foreign fiber image with the background extracted.

\section{Image Enhancement Model}

It is not difficulty to get the conclusion from the analysis result of the gray-level histogram of background-subtracted foreign fiber image that the gray-level of the background-subtracted foreign fiber image still exists in the low level part, as shown in Figure 4. This makes it difficult to perform the image segmentation to obtain the region of interesting. To solve this problem, we need to increase image contrast ration by image enhancement, so as to provide better image information basis for subsequent image segmentation. From Figure 6 we can see that the gray-level values of most pixels are below 0.08, which belongs to background figure, whereas pixels with gray-level value above 0.1 belongs to other parts of foreign figure image. And the pixels whose gray-level values are among 0.08-0.1 belong to the co-existing part of background and foreign fiber, that is, the gray edge of object and background, which exerts the most significant influences on subsequent image segmentation. Thus, a piecewise linear transform model was proposed, and the contrast of the image can be remarkably enhanced by this model, especially in the range from 0.08 to 0.1 .

A piecewise transform model splits the distribution range of image pixels into two or more pieces, and performs transformation to each piece respectively to enhance the region of interesting. In our research, the main objective is to segment the foreign fiber objects out of the background, so a three-piece nonlinear model was proposed and described as follows.

Denote the original gray level in image position (i, j) to be GO $(i, j)$, and the corresponding enhanced gray level to be GE (i, $\mathrm{j}$ ). The gray-scale range need to focus on the image enhancement is [Ll, Lh]. The three-piece linear transform model for image enhancement is defined as

$$
G E(i, j)=\left\{\begin{array}{lc}
G O(i, j) & G O(i, j) \leq L_{l} \\
8 * G O(i, j) & L_{l}<G O(i, j)<L_{h} \\
0.5 & L_{h} \leq G O(i, j)
\end{array}\right.
$$

According to the histogram analysis, we set $\mathrm{Ll}=0.078$ and $\mathrm{Lh}=0.1$. The line of this three-piece enhancement model is shown in Fig.5. 


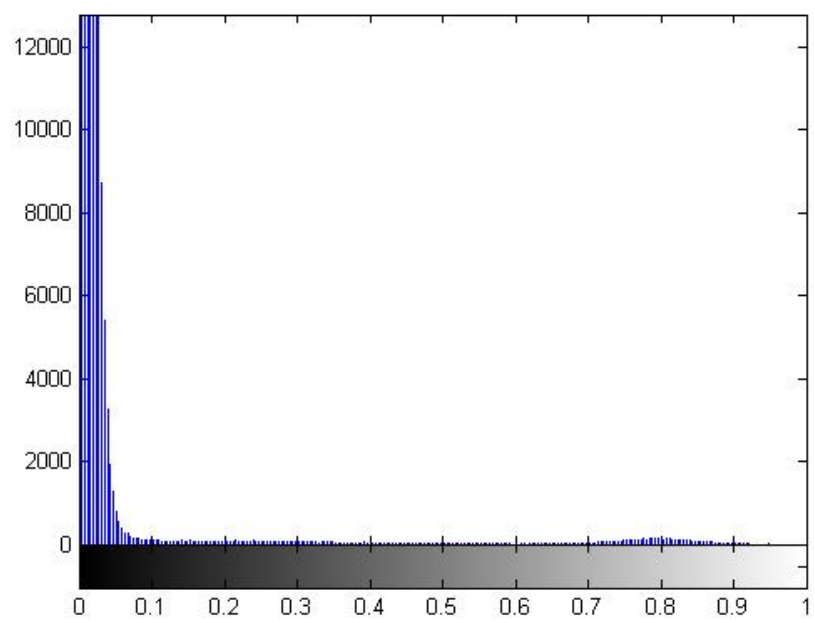

Fig. 4. Histograms of hair image

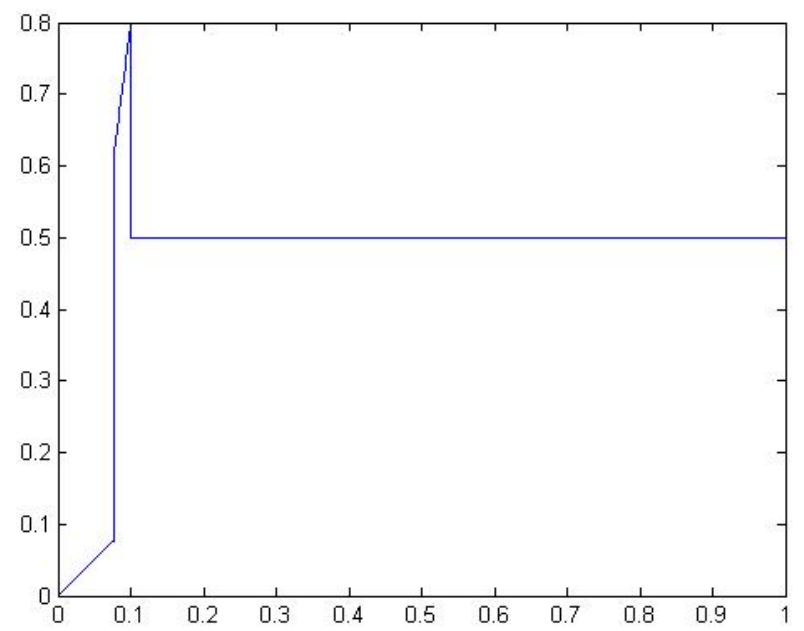

Fig. 5. Line of three-piece linear enhancement model

\section{Image Segmentation}

Speed and accuracy of an algorithm are key factors for the online visual inspection system (Golnabi and Asadpour, 2007). Hence, in addition to ensuring the segmentation accuracy, algorithms with faster speed are more attractive. The edge-based segmentation algorithm is effective when processing images with sharp edge and high contrast (Zhang, 2005). There are very little foreign fibers in lint, and the foreign fibers will be smashed by the opening machine before image acquisition. Therefore, the 
images of foreign fibers are of low contrast and are not suitable for using the edge-based segmentation algorithm. The special segmentation methods are also not fit for processing the foreign-fiber images due to their complexity and time consuming feature. Thresholding, a region-based segmentation technique, is then the preferred technique in segmenting the foreign-fiber images for its simplicity, speediness and stability.

The identification of cotton foreign fibers is carried out through real-time monitoring. However, different circumstances will have different light intensity. Therefore, the target segmentation for foreign fibers can not use a fixed thresholding value. The optimal threshold can be determined through a variety of ways, including histogram bimodal method (also known as the mode method), Otsu method and the iterative threshold method, etc. The Otsu's method (Otsu, 1979), which is also called maximum between-group variance method, is a kind of adaptive thresholding method which has the optimal threshold according to the statistics. The image is divided into two parts, namely, background and object, according to the gray character of the image. Computerizing between-group variance of background and object separated by every threshold value, we could find that when the between-group variance is the largest, the correspondent variance is optimum. The larger the variance is, the greater the difference of the two parts is. Therefore, the segmentation that has the largest between-group variance manifests the slightest chance of segmentation mistake, i.e., has the best threshold value in the sense of statistics. In this case, the dynamic thresholding was automatically selected by Ostu's method.

\section{Results and Discussion}

Seven typical foreign fibers, namely, plastic film, feather, polypropylene twine, hair, color thread, hemp rope and cloth piece, were used in the experiments. Ten samples were prepared for each type of foreign fibers. That is to say, there are totally 70 foreign-fiber samples. Adequate pure lint without foreign fibers was also prepared for making the lint layer. In our experiments, the pure lint with one type of foreign fiber dropped onto the surface at each time interval firstly was fed into the opening machine and then made into uniform thin layer, with $400 \mathrm{~mm}$ in width and $2 \mathrm{~mm}$ in thickness. The foreign fibers in the lint layer were presented in three forms, namely sheet, wirelike and villiform.

All the results of this paper were processed by the computer with the programming tools developed in Matlab7.0. Operating environment consists of: Inter Core2. PC-frequency: 2.60GHz, 2G Memory. and Windows XP was selected as the operation system.

\subsection{Analysis of Image Background Subtraction}

Foreign fiber image has low contrast ration and heterogeneous gray-level background, which brings a lot of troubles to the subsequent image processing. Therefore, we resort 
to the method background subtraction to solve to problem. Owing to the fact that foreign fiber image includes three types, i.e., sheet, wirelike, villiform, and the latter two are abundant in all foreign fibers, whereas the sheet fibers have a comparative low percentage. In addition, the connected area occupied by wirelike and villiform is so small that the foreign fiber image could be removed by erosion of smaller structural elements to get better background. On the other hand, because connected area occupied by the foreign fiber image of sheet type is comparatively large, so we need to use the large structural elements to corrode image. If we do so, the difference between the background figure subtracted and the original image will be too great to eliminate the influence of heterogeneity of background image. We can see from the gray-level analysis that the foreign fiber of sheet type mainly includes three subcategories as plastic film, feather and cloth piece, which all have deeper image gray-level and greater difference from the background figure. According to this, we could eliminate the remains of the object figure in the background by the Image gray-level correction, in order to get better background figure.

As mentioned above, the structural element employed In the process of image corrosion is round structural element with the radius of 3 . After the corrosion of foreign fiber image, we get a first-stage image background as shown in Figure 6.
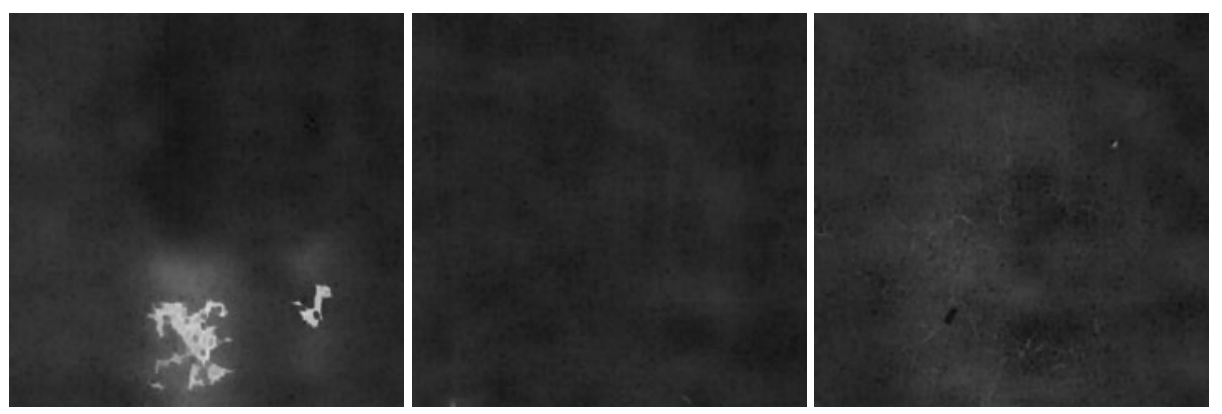

Fig. 6. Acquired background image examples: (a) background of plastic film, (b) background of hair, and (c) background of hemp rope

It is safe to say from Fig.6 we can get background figure with comparative high quality by applying the above methods to the two kinds of foreign fiber image---wirelik and villiform, but we confront problems with the sheet foreign fiber image. Thus, we could eliminate the remains of the object figure in the background by the Image gray-level correction, This paper utilized fixed threshold value 0.6 as a basic point to conduct image gray-level correction on image, that is, to replace the gray-level of all pixels that above 0.6 by 0 . Then we get a more satisfying background figure through image gray-level correction. Subsequently, subtracting origin foreign fiber image with the obtained background figure, we get the foreign fiber image with the background extracted, as shown in Figure 7. 

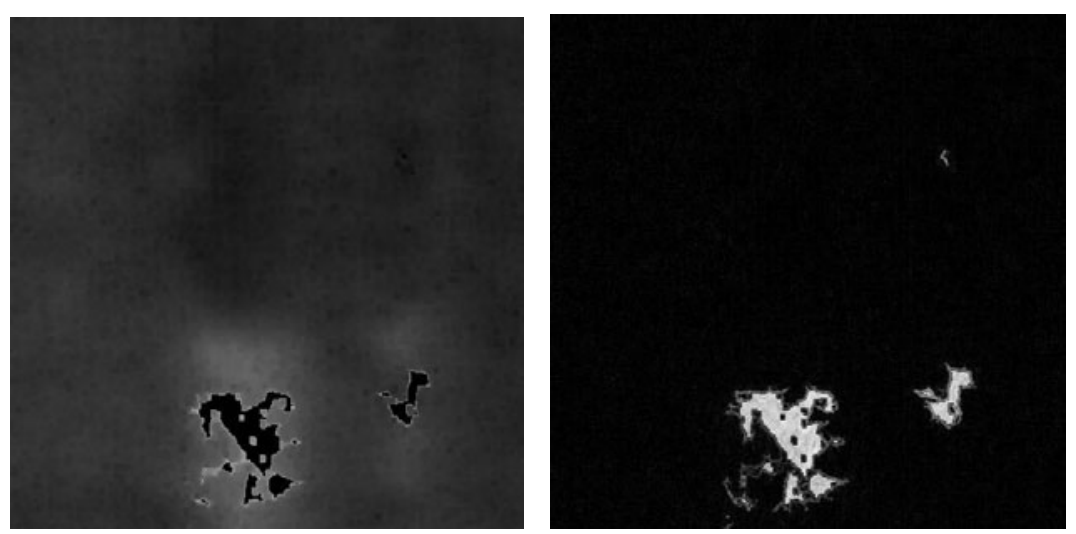

Fig. 7. (a) Acquired background image of plastic film after Image gray-level correction, (b) image of plastic film after background subtraction

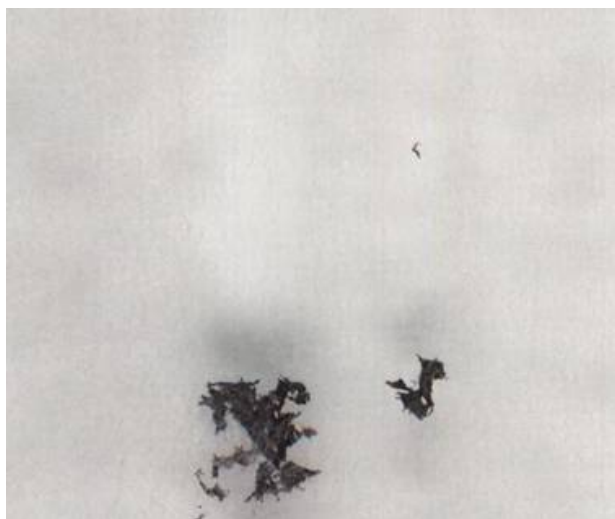

(a)

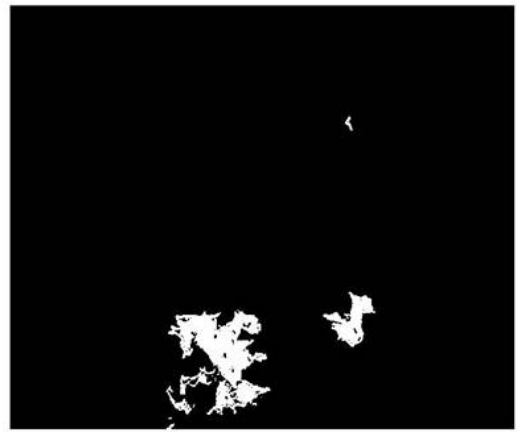

(c)

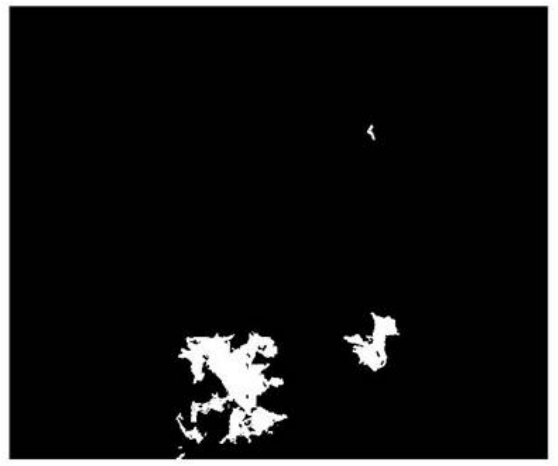

(b)

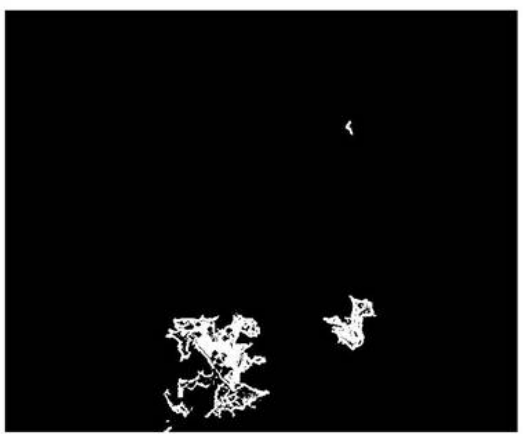

(d)

Fig. 8. (a) original image of plastic film

(b) Image of plastic film after background subtraction when the threshold is 0.5

(c) Image of plastic film after background subtraction when the threshold is 0.6

(d) Image of plastic film after background subtraction when the threshold is 0.7 
In the course of image gray-level correction, the confirmation of threshold value is crucial step. Improper choice of threshold value---too high or too low, will exert negative influence on the details of object figure in the image whose background has been subtracted. Trough several comparison of experiment results, we determine on the threshold value 0.6. Figure 8 is the foreign fiber image got from plastic film image after its background subtracted and processed by the image gray-level correction by different threshold values. From the Figure 8 it is fair to say that threshold value 0.6 can reserve the details of origin image better.

\subsection{Analysis of Image Segmentation Results}

In the paper $<$

A Fast Segmentation Method for High-resolution Color Images of Cotton Foreign Fib ers $>$ ( Zhang et al., 2011 ), the author presents a fast approach for segmenting images of foreign fibers in cotton.。 Firstly, color images were captured, and the edge of color images were detected by edge detection method which is based on improved mathematical morphology. Then the color images were converted into a gradient map, the law of experience values was analyzed, and the best thresholding of the gradient map was chosen by selecting the best experience value iteratively. The proposed method can segment the high-resolution color images of cotton foreign fibers directly and precisely, and the speed of image processing is double more than traditional methods (referred to as zhang's algorithms hereinafter).

In our research, more than 2500 sample images were tested and compared using the Otsu's algorithm, Canny algorithm, the conventional watershed algorithm and zhang's algorithm. The image segmentation results were shown as figure 9.

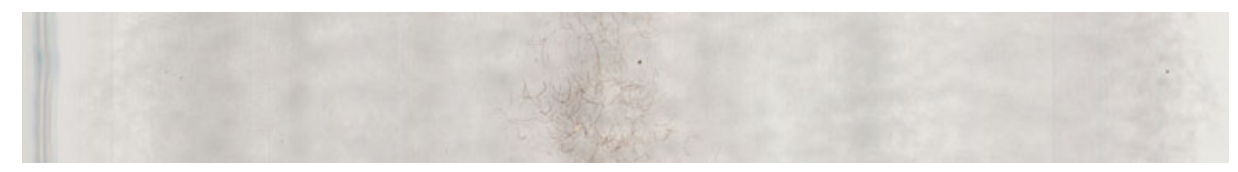

(a)
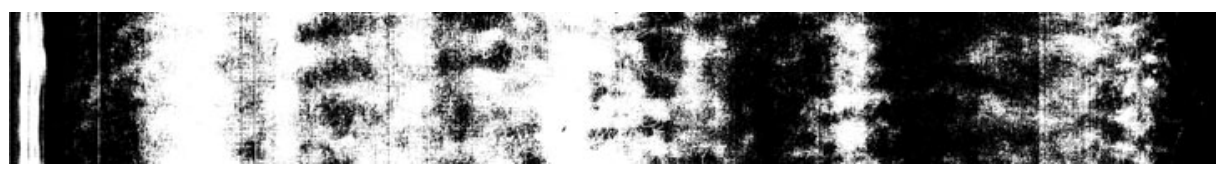

(b)

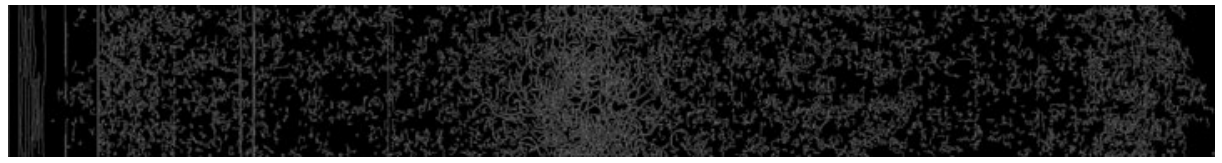

(c)

Fig. 8. (a) original image of hemp rope (b)Ostu's algorithm $\quad$ (c) canny algorithm (d) the conventional watershed algorithms (e) zhang's algorithms (f)algorithm of this paper 


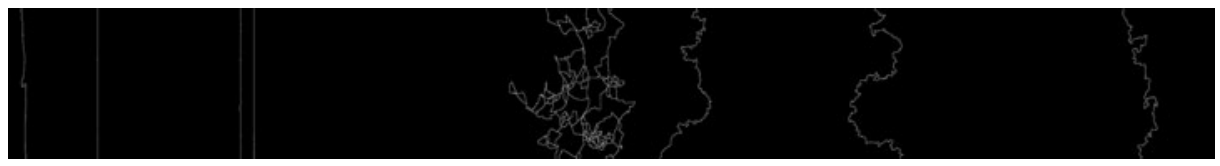

(d)

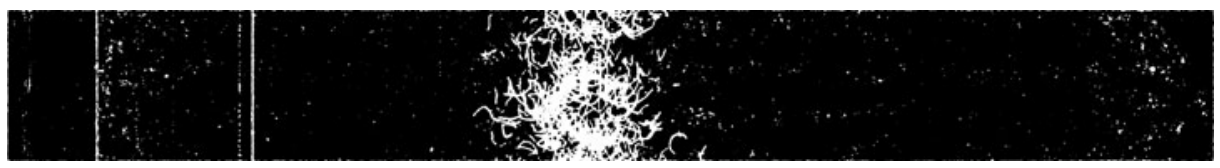

(e)

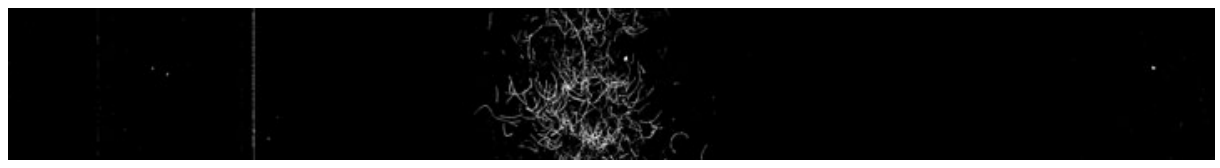

(f)

Fig. 9. (continued)

We can obviously see from the above figure the direct use of Ostu, Canny and the conventional watershed algorithms to segment image can not result in the clear segmentation of the object figure to meet our requirement. While Zhang's algorithms could get better segmentation result, the algorithm of this paper reserves more foreign fiber image details that make the segmentation result much clearer and higher segmentation accuracy.

\subsection{Analysis of Image Calculating Speed}

In our experiments of image segmentation, we have drew a comparison of the calculating speed of five methods, namely, Otsu's algorithms, Canny algorithms, the conventional watershed algorithms, zhang's algorithms and the algorithm of this paper.The results have shown that the average time for the five kinds of segmentation method of processing foreign fibers are: $0.26 \mathrm{~s}, 5.84 \mathrm{~s}, 9.23 \mathrm{~s}, 3.92 \mathrm{~s}$ and $2.38 \mathrm{~s}$. Table 1 shows the average time of the five kinds of segmentation algorithm.

Table 1. Comparison of experiment results

\begin{tabular}{cccccc}
\hline \multirow{2}{*}{ time(s) } & \multicolumn{5}{c}{ Segmentation method } \\
\cline { 2 - 6 } & Otsu & Canny & watershed & zhang's & this paper \\
\hline $\begin{array}{c}\text { average time of } \\
\text { calculation }\end{array}$ & 0.26 & 5.84 & 9.23 & 3.92 & 2.38 \\
\hline
\end{tabular}

The results shown in Table 4 indicate that, the calculating speed of Canny algorithm, the conventional watershed algorithm and Zhang's algorithm for segmentation of images for foreign fibers are significantly slower than the one presented in this paper. Moreover, seen from the segmentation result, it is obvious that this algorithm has great advantages over other algorithm in segmentation accuracy. Exclusive use of Ostu 
algorithm without any other pre-processing to segment the image, though fast in speed, could not provide us with the result we expect.

Thus, the results of above analysis showed that we can acquire better segmentation results, whether in the case of wirelike, the villiform or the sheet foreign fibers. And the calculating speed in this stage has been improved Significantly, compared with other algorithms.

\section{Conclusion}

Owing to the fact that Ostu algorithm is faced with big problems in segmenting the image that has low contrast ratio between object figure and background, small area of object figure or heterogeneous gray-level of background image---which type that foreign fiber image is unfortunately belongs to, we seek for solution by a series of pre-processing methods such as image block, background subtraction, gray balance, etc, to get better segmentation result.

The experiment result demonstrates that the algorithm of this paper far outweigh other algorithms in the past both in accuracy or speed.

The mandate of speed is a key factor for the online visual inspection system. Hence, in addition to ensuring the segmentation accuracy, algorithms of faster speed is now being studied.

\section{References}

1. Abouelela, A., Abbas, H.M., Eldeed, H., Wahdam, A.A., Nassar, S.M.: Automated vision system for localizing structural defects in textile fabrics. Pattern Recognition Letters 26(10), 1435-1443 (2005)

2. Bakker, T., Wouters, H., van Asselt, K., Bontsema, J., Tang, L., Muller, J., van Straten, G.: A vision based row detection system for sugar beet. Computers and Electronics in Agriculture 60(1), 87-95 (2008)

3. Chaira, T., Ray, A.K.: Threshold selection using fuzzy set theory. Pattern Recognition Letters 25(8), 865-874 (2004)

4. Chen, Y.R., Chao, K.L., Kim, M.S.: Machine vision technology for agricultural applications. Computers and Electronics in Agriculture 36(2-3), 173-191 (2002)

5. Funck, J.W., Zhong, Y., Butler, D.A., Brunner, C.C., Forrer, J.B.: Image segmentation algorithms applied to wood defect detection. Computers and Electronics in Agriculture 41(1-3), 157-179 (2003)

6. Zhang, H., Frittsb, J.E., Goldmana, S.A.: Image segmentation evaluation: A survey of unsupervised methods. Computer Vision and Image Understanding 110, 260-280 (2008)

7. Kim, B.G., Shim, J.I., Park, D.J.: Fast image segmentation based on multiresolution analysis and wavelets. Pattern Recognition Letters 24(6), 2995-3006 (2003)

8. Kwon, S.H.: Threshold selection based on cluster analysis. Pattern Recognition Letters 25(9), 1045-1050 (2004)

9. Lieberman, M.A., Bragg, C.K., Brennan, S.N.: Determining gravimetric bark content in cotton with machine vision. Textile Research Journal 68(2), 94-104 (1998)

10. Millman, M.P., Acar, M., Jackson, M.R.: Computer vision for textured yarn interlace (nip) measurements at high speeds. Mechatronics 11(8), 1025-1038 (2001) 
11. Otsu, N.: A threshold selection method from gray-level histograms. IEEE Transactions on SMC 9(1), 62-66 (1979)

12. Pichel, J.C., Singh, D.E., Rivera, F.F.: Image segmentation based on merging of sub-optimal segmentations. Pattern Recognition Letters 27(10), 1105-1116 (2006)

13. Susomboon, R., Stan Raicu, D., Furst, J.: A hybrid approach for liver segmentation. In: MICCAI 2007 Workshop Proceedings of the 3D Segmentation in the Clinic: a Grand Challenge, pp. 151-160 (2007)

14. Su, Z.W., Tian, G.Y., Gao, C.H.: A machine vision system for on-line removal of contaminants in wool. Mechatronics 16(5), 243-247 (2006)

15. Yang, W., et al.: A new approach for image processing in foreign fiber detection. Comput. Electron. Agric. (2009), doi:10.1016/j.compag.2009.04.005

16. Zhang, H., Fritts, J.E., Goldman, S.A.: Image segmentation evaluation: a survey of unsupervised methods. Computer Vision and Image Understanding 110, 260-280 (2008)

17. Zhang, X., et al.: A Fast Segmentation Method for High-resolution Color Images of Cotton Foreign Fibers. Computers and Electronics in Agriculture (2011), doi:2011.06.005

18. Zhang, Y.J.: Image Engineering(II)Image Analysis. Tsinghua University Press, Beijing (2005) 